\title{
A NOVEL PROJECTION ALGORITHM FOR PRODUCTION LAYOUT EXTRACTION FROM POINT CLOUDS
}

\author{
Marek Bureš**, Sergo Martirosov, Jiří Polcar \\ University of West Bohemia, Regional Technological Institute, Univerzitni 8, 30614 Pilsen, Czech Republic \\ * corresponding author: buresm@rti.zcu.cz
}

\begin{abstract}
The paper is focused on point cloud data processing obtained by 3D laser scanning. The scanning devices are at a very advanced level and after reaching their possible maximum scanning speeds, manufacturers are now more focused on a minimization of the devices. However, there is still a lack of software solutions for a simple and successful model creation from point cloud data or data evaluation. This paper briefly describes the laser scanning principle and the process of production floor layout capturing. Furthermore, a newly developed algorithm for an extraction of specific areas of point cloud is introduced. The algorithm was tested and compared with other solutions for a production layout development. After testing, the standalone software application called CloudSlicer ${ }^{\mathrm{TM}}$ was programed and the user interface is also presented.
\end{abstract}

KEYWORDS: 3D laser scanning, point cloud, production layout, projection algorithm.

\section{INTRODUCTION}

Point cloud is the type of data that is produced by $3 \mathrm{D}$ laser scanners that measure a large number of points on the external surfaces of objects around them. For this work, a terrestrial laser scanner was used. These devices are mainly used in geodesy [1], architecture and urbanism [2 4], archaeology [5] or, for example, a crime scene reconstruction [6. Another developing field for the laser scanning is the manufacturing, specifically production floor planning and management. Laser scanning in this field is used mainly for production floor layout capturing [7, 8]. Some applications can also be found in the field of virtual ergonomics and workplace evaluation [9, 10.

The laser scanning technology brought a great potential for production floor layout capturing. The method is very accurate, fast and when used correctly, reduces the error rate caused by the human factor. It can play a very important role in the upcoming Industry 4.0 and smart factories, where fast and precise data acquisition will be one of the key problems. These days, scanning devices are technologically advanced, as the scanning speed of new scanner types can be up to 1000000 points per second (pts/sec). Furthermore, the simplification and reduction of device dimensions are gradually being made, as the efficiency parameters reach their maximum levels, mostly, these parameters can be altered to increase the competitiveness. For example, the recently introduced device BLK360 [11] from Leica Geosystems has a very suitable combination of parameters - satisfactory scanning speed and accuracy, small dimensions and a low purchase price.

Even though devices are efficient, there is a problem with the further data processing. Although devices can produce high quality models as seen in Figure 1 , a more autonomous processing of models is missing.
Software applications that are currently available have at least one of the following disadvantage:

- Software is not optimized enough for displaying sufficient amount of points.

- Software does not support point clouds at all.

- Software is able to only display point clouds but not use them as binding.

- Software does not provide algorithm parameterization.

In addition, most of the very accurate devices use majority of the algorithms made for point clouds. Use of these algorithms on point clouds that are acquired by a terrestrial laser scanner causes either failure of the algorithms or does not provide outputs that are good enough (Figure 2).

\subsection{D LASER SCANNING PRINCIPLE}

According to [12], the principle on which the TLS (Terrestrial laser scanner) and LiDAR/ALS (Light Detection and Ranging) scanning devices operate, is the same. The term LiDAR comes from combining the words light and radar. The principle of LiDAR (Light Detection and Ranging) scanning devices is the projection of a laser beam on the scanned object 13 . This beam is then reflected back to the device; and, based on the time it takes a projection to reach back; the distance between the origin of the device and the point on the object is calculated. The device is designed in such a way that it can rotate its turret by $360^{\circ}$ around the horizontal axis and the mirror with the camera by $360^{\circ}$ around vertical axis. Based on both the rotation angles and the distance of the object to be scanned, with the use of a triangular method, the location of the point in space is defined. With the scanning speed of $360000 \mathrm{pt} / \mathrm{s}$, for example, the process of the beam 


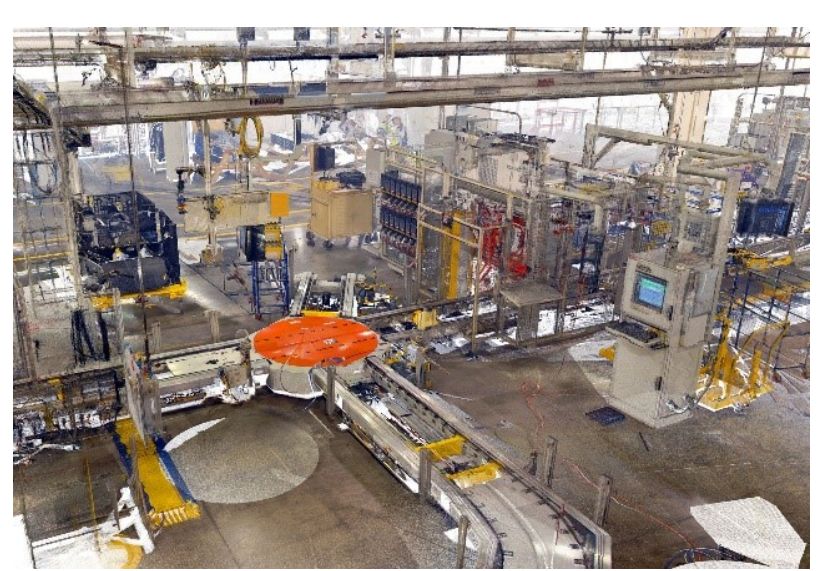

Figure 1. High-resolution industrial point cloud.

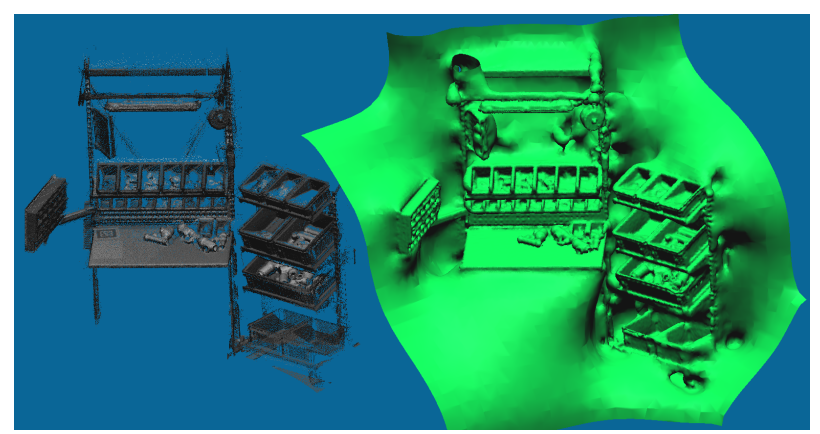

Figure 2. Left - point cloud model; Right - Failed algorithm rendering.

projection and reflection and the following location calculation, repeats 360000 times per second (after each location definition, device components rotate for a very small portion around horizontal and vertical axes and the procedure is repeated again until completely finished).

The LiDAR measurements are based on a general laser range equation that is defined by Eq. 1 as follows [12]:

$$
P_{R}=\frac{P_{T} D^{2} \sigma}{4 \pi R^{4} \beta_{t}^{2}} \eta_{A t m} \eta_{S y s}
$$

where: $P_{R}$ - received signal power $[W], P_{T}$ transmitter power $[W], \sigma$ - effective target cross section $\left[m^{2}\right], R$ - system range to target $[m], D$ - receiver aperture diameter $[m], \beta_{t}-$ the laser beam width $[-], \eta_{\text {Atm }}-$ atmospheric transmission factor $[-], \eta_{\text {Sys }}$ - system transmission factor $[-]$.

Following 12, the relation between the laser beam width $\left(\beta_{t}\right)$, aperture illumination constant $\left(K_{a}\right)$, wavelength of laser light $(\lambda)$ and aperture diameter $(D)$ is given in Eq. 2 .

$$
\beta_{t}=\frac{\left(K_{a} \lambda\right)}{D}
$$

The effective target cross-section (also known as backscattering cross-section) $\sigma$ is defined 12 by Eq. 3

$$
\sigma=\frac{4 \pi}{\Omega} \rho A_{s}
$$

where: $\Omega$ - scattering solid angle of the target $[s r], \rho$ - target reflectance $[-], A_{s}$ - target area $\left[m^{2}\right]$.

Compared to LiDAR measurements, when scanning with TLS, the reflecting surface exceeds the laser footprint. The scanned object is subsequently termed an extended target. This aspect changes the general laser range equation Eq. 1 .

According to [12, the function value of inverse range $1 / r^{4}$ is replaced in the laser range equation with the value $1 / r^{2}$. The equation is further simplified based on the Lambertian properties of a target. With respect to a perfect Lambertian target, the backscatter power mainly depends on a target reflectivity $\rho$, angle of incidence $\alpha$, and range to the target $\mathrm{R}$ as given in Eq. 4 .

$$
P_{R}=\frac{\pi P_{E} \rho \cos \alpha}{4 r^{2}} \eta_{A t m} \eta_{S y s}
$$

where: $P_{R}$ - detected signal power, $P_{E}$ - transmitted signal power, $\alpha$ - angle of incidence, $\rho$ - reflectance of a material, $\eta_{\mathrm{Atm}}$ - atmospheric transmission factor, $\eta_{\text {Sys }}$ - system transmission factor, $r$ - range.

This version of a "laser equation" is commonly used in the usage of the TLS in civil engineering.

\subsection{PRODUCTION FLOOR LAYOUT SCANNING}

Individual steps of the process are described in [14]. When it's possible to scan a factory hall (in some cases because of dimensions, production fluency or safety, scanning of a layout is impossible), the positions at which the data will be collected are defined. At each of these positions, one individual scan of the space is done. Then, the device is moved to the next position and the process is repeated until the whole object (building, machine, etc.) is scanned. Next, the acquired data are processed with the use of a special software (Leica Cyclone, Autodesk ReCap) and then assembled into a complete 3D point cloud model. That model can be used for an additional processing, but current software packages lack the capability to do so.

\subsection{Point ClOUd USE IN VIRTUAL REALity}

The projection of the point cloud into virtual reality devices offers the possibility of a very realistic perception. This can also be used for production floor layout planning, where a team of engineers can have a realistic walk through the factory from different locations. They can also work on the project at the same time.

There are different possibilities how to visualize the point clouds in a virtual reality. Very common is a classical stereoscopic projection (Figure 3 ) but in these days, the top experience from a virtual reality is with headsets.

The point cloud data can also be used for designing $3 \mathrm{D}$ models for virtual reality. Figure 4 shows point cloud data of Regional Technological Institute (RTI) manufacturing hall. This point cloud was used to recreate a $3 \mathrm{D}$ model that was constructed with Recap 


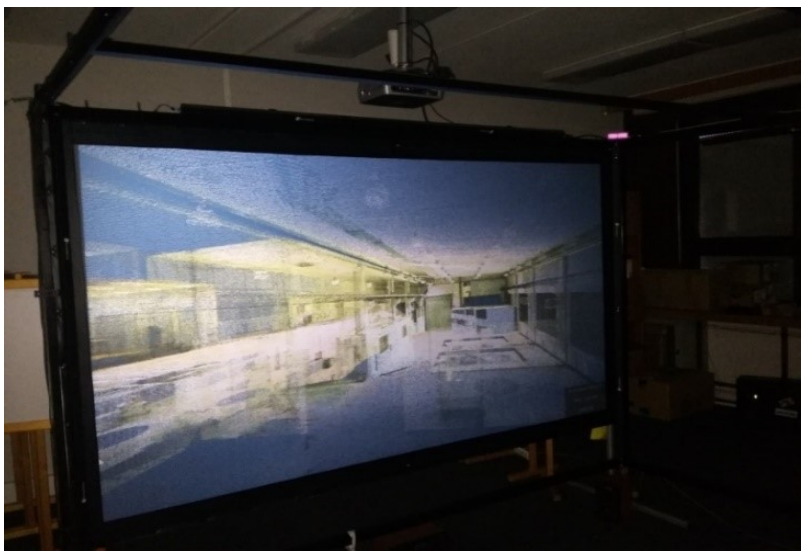

Figure 3. CAVE point cloud projection.

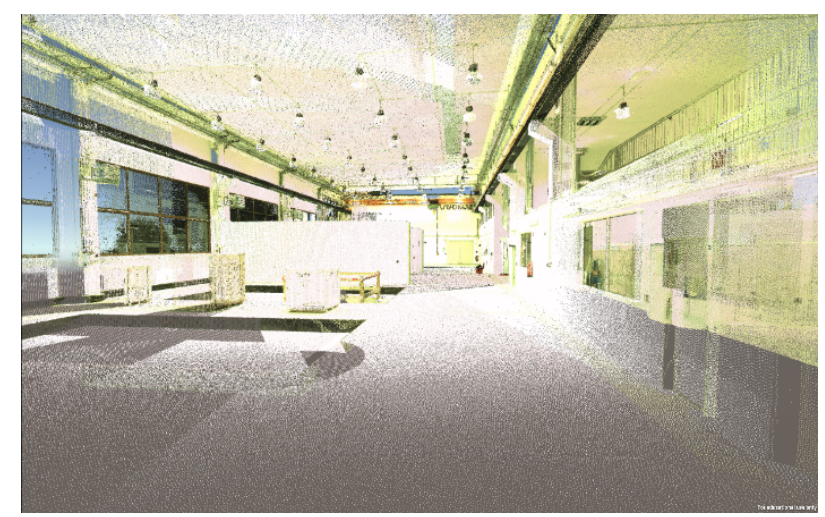

FiguRE 4. RTI hall point cloud only.

and exported to Unity 3D Game Engine. For now, the model is used as a virtual walkthrough in the hall to show the current state of the real hall. If the layout of the hall changes, it is possible to re-scan the environment and with the use of a specialized software (Cyclone), remove the old data from the main point cloud and add the new one.

In the project, we included both the 3D model and the cloud data of the hall itself so that viewers who go through the virtual reality hall could switch between views and see the difference/similarity between the point cloud and the 3D model (Figure 5.

\section{ReSEARCh Aim}

With the use of the point cloud data for a production floor layout modelling [14, no available solution for an easy layout extraction was found. Previous researches in this area focused, for example, on pipeline systems [15], buildings reconstruction [16, 17, segmentation method for 3D modelling from point clouds [18] or on complex studies [19]. All these methods have a one combined requirement, a high resolution of the point cloud (high point density in point net). The mentioned methods are also difficult to process and require a high performance hardware.

Furthermore, there are multiple studies in this field regarding the point cloud data extraction. The Automated $3 \mathrm{D}$ reconstruction of multiple-room building

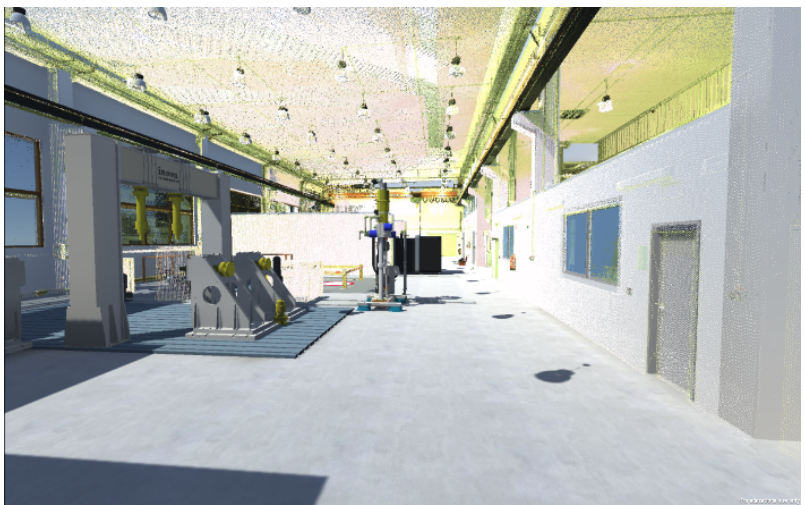

Figure 5. RTI hall point cloud with a 3D Model.

interiors in point clouds 20] uses a special algorithm to detect hollow parts of the BIM (Building Information Model), such as doors and windows [20]. The filtered point clouds were projected onto a binary map in order to trace the floor-wall boundary, which was further refined through subsequent segmentation and regularization procedures. A similar algorithm for an accurate detection of wall and openings was described by [21]. The authors have presented an automatic method for reconstructing room information from raw point cloud data using only $3 \mathrm{D}$ point information. The [22] focused on an automatic reconstruction of a volumetric, parametric building model from indoor point clouds resulting in $3 \mathrm{D}$ models for an architectural software. They proposed a novel reconstruction method in which the representation of buildings using parametric, interrelated, volumetric elements is an integral component and focused especially on wall connectivity and wall thickness.

Another area is the segmentation of point clouds described, for example in, 23. The authors proposed a novel 3D segmentation framework, RSNet (Recurrent Slice Network), to efficiently model local structures in point clouds. The 24 focused on a semi-automated building facade footprint extraction that uses image segmentation to extract contour areas, which contain facade points of buildings, trees and other objects. The proposed method first generates a georeferenced feature image from the mobile LiDAR data, thus transforming the problem of understanding point clouds into that of understanding images. The extracted facade footprints are not only beneficial for the facade reconstruction but are also meaningful for the segmentation of building point clouds.

Most of these methods focus on perimeter walls and identify interiors (furniture and other objects) as a clutter and separate it. For industrial layout detection are these objects however essential. Due to these reasons, we aimed at a new algorithm development which will ease the point cloud processing for $2 \mathrm{D}$ industrial layout creation purposes. Also, having a simple, user friendly software application was another goal. The main functionality of the new algorithm, respectively new software, is the projection of the 
selected points to the plane. The name for the software is CloudSlicer ${ }^{\mathrm{TM}}$ as it describes the main functionality.

\section{Results}

\subsection{Point ClOUd PROJECTION ALGORIThM}

As the name CloudSlicer ${ }^{\mathrm{TM}}$ suggests, the main purpose of the algorithm is a "slicing" of point cloud. Point cloud files usually contain the following information for each point: X, Y, Z, R, G, B and I - where the first three letters define Cartesian coordinates of the point, the second three letters define colour and I is the intensity value of the laser beam for each point. The intensity (a dimensionless variable) is defined as a product of reflectance of the material. Such data can be obtained by converting the proprietary scanning device data to an exchange format, usually in form of ASCII files. The first step of the algorithm is to transfer the ASCII file into a binary format. The reason of the transfer is that the binary reading is much faster compared to text file reading. The speed of reading binary files is much higher than parsing ASCII files. Speeding this process up is not necessary, but very convenient, as the data will usually need to be read multiple times from the file, which is often too big to fit in the workstation's working memory. The next step is to define $\mathrm{X}$ and $\mathrm{Y}$ axes in the point cloud space, which will correspond to the horizontal and vertical axes of the final image. Another axis is the $\mathrm{N}$ axis, which will define the direction of the normal. This will be the projection direction of the point clouds to their underlying pixels in the final image. By default, all three axes are perpendicular. Last two inputs are float values, which represent near and far culling distances. This will practically define the transformation function of the point cloud points into the resulting image.

When a point from the point-cloud is loaded, it will be the input for the transformation function. The result of this function is the $\mathrm{x}$ and $\mathrm{y}$ coordinates of the bitmap pixel and further values that will eventually define the colour of the pixel. The pixels' $\mathrm{x}$ and $\mathrm{y}$ coordinates are computed from the distance of planes defined by $\mathrm{XN}$ and $\mathrm{YN}$ vectors. If the resulting coordinates do not fit in the interval between zero and the bitmap size or the culling distances, it will be skipped. The flow diagram of the CloudSlicer ${ }^{\mathrm{TM}}$ algorithm is visualized in Figure 11

The resulting colour of the pixels in the bitmap image can be computed in different ways. By default, it is the average colour from the points above the pixel (in direction of the $\mathrm{N}$ vector, by averaging RGB values). Or, it can be the greyscale values representing the average height of the points above the pixels, resulting in a height map. These functions can be further extended.

Finally, the raw data are exported in the BMP bitmap picture file format, which can be compressed and converted to JPG or PNG formats, which can be

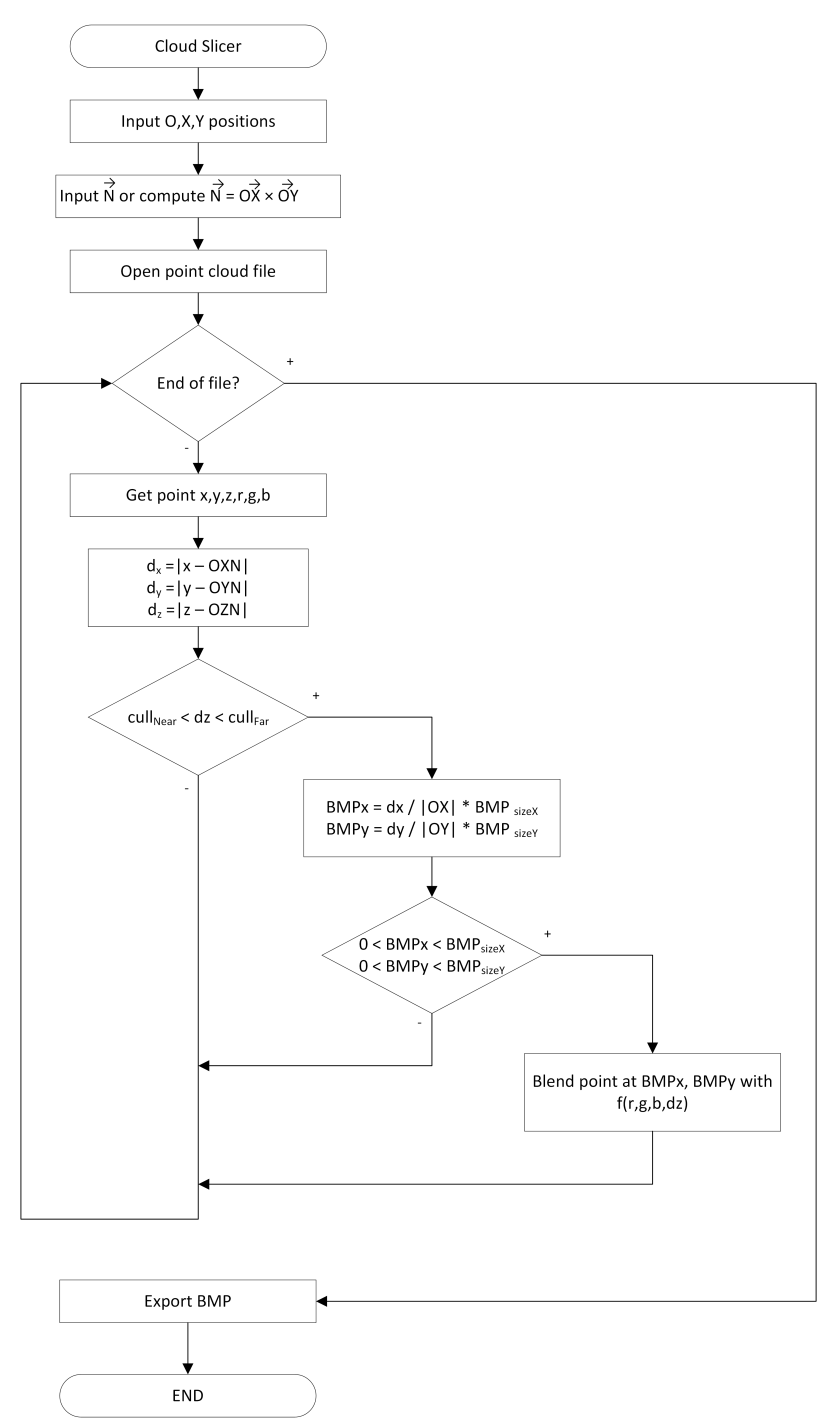

Figure 6. Flow diagram of the CloudSlicer algorithm.

imported in most CAD programs or layout designing tools. Examples of such projected point cloud of a factory hall can be seen in Figure 6 .

\subsection{Algorithm VAlidation on Layout MODELLING}

Algorithm described above was validated by the production floor layout modelling procedure. The whole procedure consists of two parts - data acquisition and data processing. Firstly, the layout analysis was made in order to determine:

- if the layout can be scanned and under which conditions,

- where will be the positions for scanning,

- and how big is the traffic intensity.

Next step was the data acquisition, described in 1.2 followed by the data computer processing in the computer. The scanning device used for this experiment was Leica ScanStation C5 with the parameters described in Table 1 . 


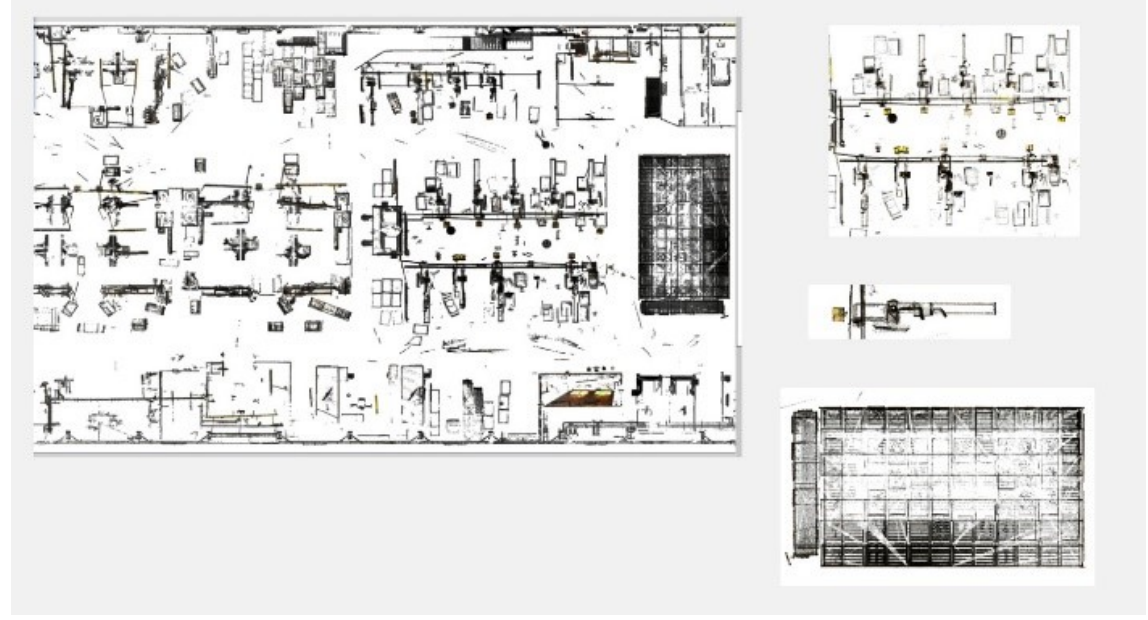

Figure 7. CloudSlicer 2D layout output.

\begin{tabular}{cc}
\hline Parameter & Value \\
\hline Quality of scan & Medium \\
Accurancy & $2 \mathrm{~mm}$ \\
Range & 2 up to 35 meters \\
Scan rate & $25000 \mathrm{pts} / \mathrm{sec}$ \\
Field of view & $2360^{\circ}$ (horizontal) $/ 270^{\circ}$ (vertical) \\
\hline
\end{tabular}

TABLE 1. Leica ScanStation C5 parameters.

The final model was then processed by two methods - the first method was focused on the use of Autodesk software, second method was focused on CloudSlicer ${ }^{\mathrm{TM}}$ algorithm and layout creation in visTable software.

In the first method, we used Autodesk ReCap software. With this software, point clouds from laser scanner could be transferred into a compatible file RCP. This file could then be used in other Autodesk software that supports the point cloud processing. In addition, using Recap, the whole model was divided into smaller parts. These parts can be stored into point cloud library for their future possible use and they can be assembled into a 2D layout in AutoCAD software or 3D layout in Inventor software. The purpose of the division into smaller components is the possibility of a spatial arrangement in the following layouts. Unfortunately, the final 2D layout in AutoCAD software was not of a good quality and also AutoCAD does not provide any layout analytic tools like material flow analysis. These tools are available only in Factory Design Suite.

In the second method, we used the visTable software for a layout creation. This software is very useful due to its simplicity, clarity and disposition of analytic tools. However, visTable cannot work with point clouds at all. That was the reason for involving CloudSlicer ${ }^{\mathrm{TM}}$. With this algorithm, a very accurate BMP 2D layout was created. This layout can be loaded straight into visTable as an underlay, or divided into smaller parts and assembled in visTable as a moveable 2D layout (Figure 7).

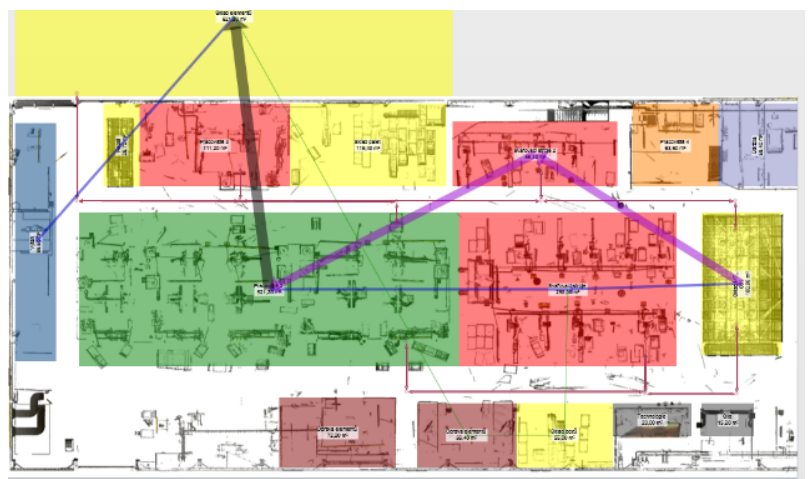

Figure 8. CloudSlicer layout with visTable material flow analysis.

The second method was then compared with a conventional way of layout capturing and modelling, which consists of direct dimension measurements (with the use of tapes or laser distance meters), followed by machine and object modelling and, finally, assembling these models into a layout in visTable software. The comparison results are in Figure 8 For the comparison, the data acquisition times (which were acquired during the factory scanning) and layout creation times (2D/3D) were monitored and then compared with the values obtained during measurements and layout modelling when using the conventional method.

The values that we found were also re-calculated for a theoretical use of the BLK360 device with a scanning speed of $360000 \mathrm{pt} / \mathrm{s}$. The data values are shown in Table2. It is clear that the scanning method 
Duration of activities for creating 2D/3D machine layout

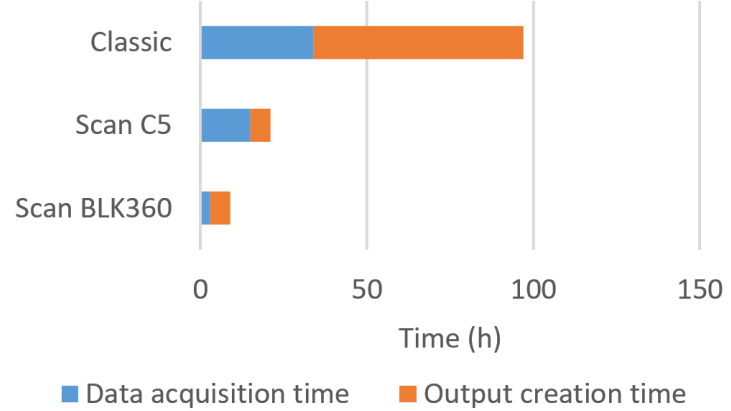

Figure 9. Process duration comparison.

is approximately five times faster compared to the conventional method. However, the scanning methods require a high performance hardware, experienced users and a special software.

\subsection{CloudSlicer ${ }^{\mathrm{TM}}$ Standalone ApplicATION}

After the validation of the CloudSlicer ${ }^{\mathrm{TM}}$ algorithm we needed to create a user friendly environment thus the standalone software application was created. The CloudSlicer ${ }^{\mathrm{TM}}$ software was created in the Unity 3D game engine. It is possible to use both PTS and CSB file formats in this software, but the CSB is recommended more as the loading time is much faster. In case there is only PTS file available from the previous software, it is possible to convert the file to the CSB format using the convert function in CloudSlicer ${ }^{\mathrm{TM}}$. Once the point cloud data is loaded, the scene is saved and the work can begin. Two main tools are available: the WallSlicer and the FloorSlicer. Both these tools are used to define a box, where the point clouds will be projected into the middle plane. They are the user interface for the definition of the X, Y, N and culling distance values for the CloudSlicer ${ }^{\mathrm{TM}}$ algorithm described in 3.1). After the definition of these boxes, a file with the definitions is generated and is processed by CloudSlicer ${ }^{\mathrm{TM}}$. When the task is done, the resulting images (slices) are placed into the scene, making it possible to replace the point clouds (or its parts) with much more efficient data.

The result of CloudSlicer ${ }^{\mathrm{TM}}$ is shown below. The spots with the yellow colour indicate that there was no point cloud data in those spots. For better results, scanning from different positions is advised. For simple objects, one scan might be sufficient but for complex objects such as the church below, it is necessary to make several scans from different angles.

\section{NeXt ADVAnCE}

Our CloudSlicer ${ }^{\mathrm{TM}}$ application is in its working stage and can already be used to fulfil its purpose, but still,

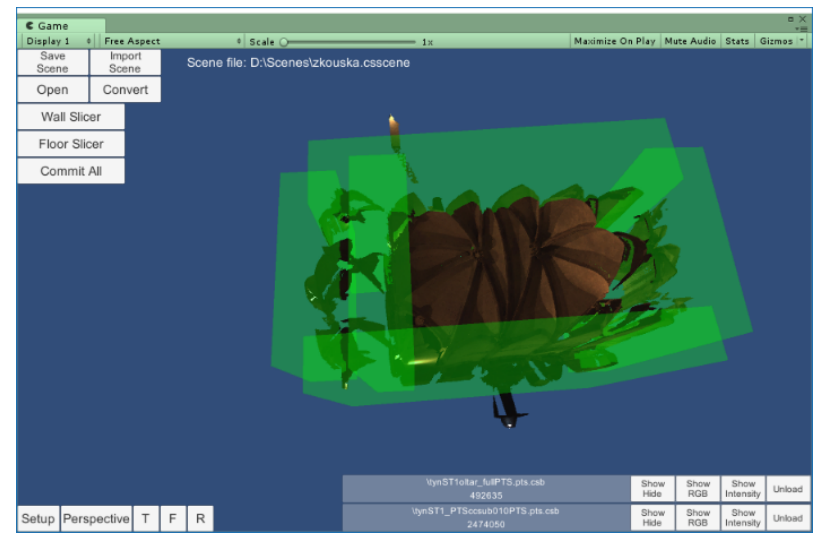

Figure 10. Slicing point cloud data in CloudSlicer ${ }^{\mathrm{TM}}$.

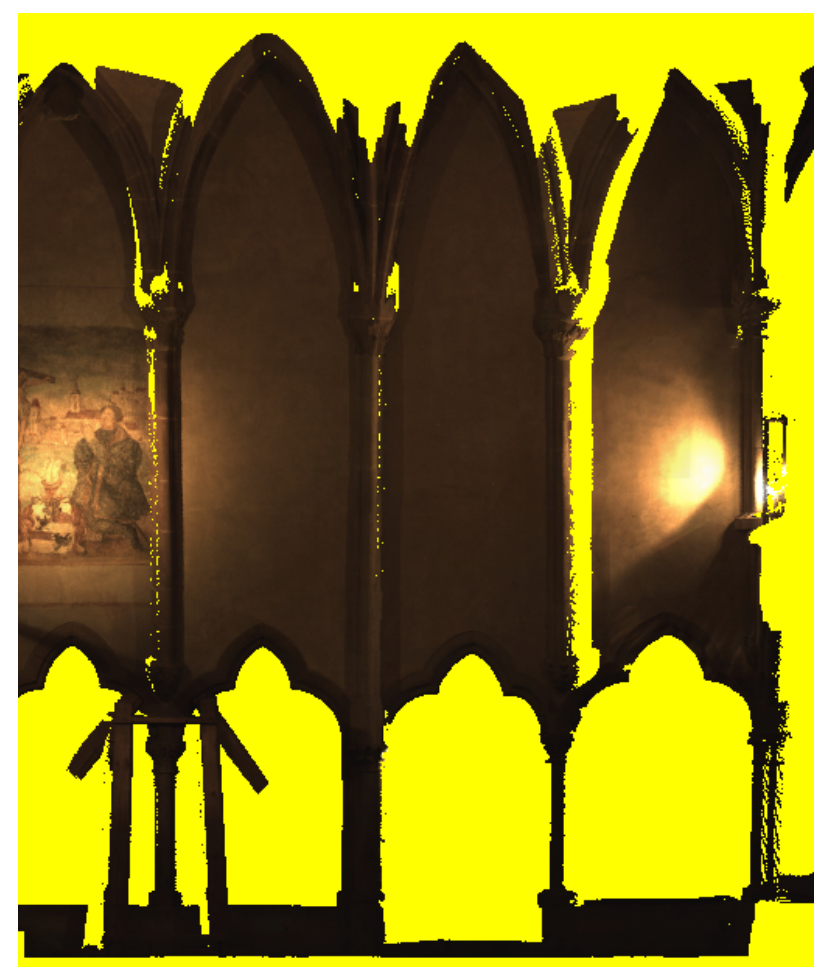

Figure 11. The example of wall slicer output.

we could implement some improvements and additional functionalities. Those could be the possibility of colour manipulation of empty spots of the images or cloud data. The possibility to slice cloud data from a perspective view, possibility to cut out some data from the point cloud, add several cloud data to the scene and merge them and etc. Next steps in the algorithm's development is definitely the research of algorithms for modelling rectangular and planar objects, which are very common in production floor layouts. These algorithms will help in production floor layout modelling and in modelling for ergonomic analyses. In the virtual reality, there will be a focus on a research of controlling and manipulating point clouds via tracking devices. 


\begin{tabular}{ccc}
\hline Output/Value & Data acquisition time $(\mathrm{h})$ & Output creation time $(\mathrm{h})$ \\
\hline \multicolumn{3}{c}{ Conventional method } \\
\hline 2D layout & 31 & 63 \\
3D layout & 34 & 63 \\
\hline \multicolumn{2}{c}{ Scanning method Leica C5 (25 000 pt/s) } \\
\hline 2D layout & 15 & 5 \\
3D layout & 15 & 6 \\
\hline \multicolumn{2}{c}{ Scanning method Scanner BLK360 (360 000 pt/s) } \\
\hline 2D layout & 3 & 5 \\
3D layout & 3 & 6 \\
\hline
\end{tabular}

TABLE 2. Values for methods comparison.

\section{Conclusion}

The aim of this paper was to introduce research results of point clouds and its use in industrial engineering. We investigated how a terrestrial scanning device can be used in industrial layout modelling and how effective this method is compared to common methods of modelling 3D models by measuring them and then using a special 3D modelling software. During the model acquisition, time values were monitored. These values show that the scanning method is approximately five times faster than the common way of measuring and modelling layouts. However, this method requires a more experienced user and a high performance hardware.

Related to the modelling process, algorithm CloudSlicer $^{\mathrm{TM}}$ was developed. This algorithm is able to create a very accurate $2 \mathrm{D}$ layout in a short period of time and with low hardware requirements. With the development of this algorithm, we tried to address some of the issues stated at the beginning of the paper. If the software for the layout modelling doesn't support the point clouds, it's hard to add new functionalities or plugins as many of those programs are being protected by the developer, so a creation of an extra application between the point clouds processing software and the layout modelling software that would serve as a bridge is needed. Thus the CloudSlicer ${ }^{\mathrm{TM}}$ was developed. Nearly every layout modelling software allows an import of standardized simple files like bitmap or vector pictures. Those can be than used as an underlay for the layout creation. Due to this fact, the decision about output (BMP picture file format) of the CloudSlicer ${ }^{\mathrm{TM}}$ application was made. So far, the CloudSlicer $^{\mathrm{TM}}$ application doesn't allow much of the parametrization. The user can now only select specific areas of the point cloud that he wants to create a projection of, but in the future, we would like to add some functionalities, such as colour parametrization. During the research of available software solutions, no software capable of an automatic creation of reasonable $3 \mathrm{D}$ models from more complex point clouds was found, so this could also be some possibility for the future development of the application as well as display or manipulation with the point clouds with the use of virtual reality devices.

We hope that this application could open new possibilities in the 3D layout and modelling creation for industrial engineering. It should combine advantages of a fast layout creation and collaborative decentralized work.

\section{ACKNOWLEDGEMENTS}

This paper was created with the subsidy of the project LO1502 - Development of Regional Technological Institute carried out with the support of Ministry of Education, Youth and Sports of the Czech Republic.

\section{REFERENCES}

[1] O. Monserrat, M. Crosetto. Deformation measurement using terrestrial laser scanning data and least squares 3D surface matching. ISPRS Journal of Photogrammetry and Remote Sensing 63(1):142-154, 2008. DOI:10.1016/j.isprsjprs.2007.07.008

[2] P. Tang, et al. Automatic reconstruction of as-built building information models from laser-scanned point clouds: A review of related techniques. Automation in Construction 19(7):829-843, 2010. DOI:10.1016/j.autcon.2010.06.007.

[3] C. Sahin, et al. Producing 3D city model with the combined photogrammetric and laser scanner data in the example of Taksim Cumhuriyet square. Optics and Laser Engineering 50(12):1844-1853, 2012. DOI:10.1016/j.optlaseng.2012.05.019

[4] H. Rüther, et al. Laser scanning for conservation and research of African cultural heritage sites: the case study of Wonderwerk Cave, South Africa. Journal of Archaeological Science 36(9):1847-1856, 2009. DOI:10.1016/j.jas.2009.04.012

[5] M. Hůlková, K. Pavelka, E. Matoušková. Automatic classification of point clouds for highway documentation. Acta Polytechnica 58(3):165-170, 2018. DOI:10.14311/AP.2018.58.0165

[6] U. Buck, et al. Accident or homicide - Virtual crime scene reconstruction using 3D methods. Forensic Science International 225(1-3):75-84, 2013. DOI:10.1016/j.forsciint.2012.05.015 
[7] K. Pukanska. The application of terrestrial laser scanning for spatial visualization of laboratories of the BERG faculty. Acta Montanistica Slovaca 17(4):341-347, 2012.

[8] E. Lindskog, et al. Visualization support for virtual redesign of manufacturing systems. In Proceedings of 46th CIRP Conference on Manufacturing Systems, vol. 7, pp. 419-424. 2013. DOI:10.1016/j.procir.2013.06.009

[9] M. Bureš, J. Polcar. Comparison of 3D Scanning and 3D Modelling of a Workplace from Various Aspects. In IEEE International Conference on Industrial

Engineering and Engineering Management, pp. 306-310. 2016. DOI:10.1109/IEEM.2016.7797886

[10] M. Hovanec, et al. Proactive ergonomics based on digitalization using 3D scanning and workplace modeling in Texnomatix Jack with Augmented Reality. Nase More 61(1-2):22-26, 2014.

[11] Leica Geosystems. Leica BLK360 Imaging Laser Scanner. https://leica-geosystems.com/products/ laser-scanners/scanners/blk360, 2017.

[12] C. Suchocki, J. Katzer. Terrestrial laser scanning harnessed for moisture detection in building materials Problems and limitations. Automation in Construction 94(2018), 2018. DOI:10.1016/j.autcon.2018.06.010.

[13] M. Jaboyedoff, et al. Use of LiDAR in landslide investigations: A review. Natural Hazards 61(1):5-28, 2012. DOI:10.1007/s11069-010-9634-2

[14] M. Strapek, P. Hořejší, J. Polcar. 3D laser scanned data processing possibilities for production floors models. In Proceedings of 28th International Business Information Management Association Conference, pp. 2920-2930. 2016.

[15] G. Pang, et al. Automatic 3D industrial point cloud modeling and recognitions. In Proceedings of 14 th IAPR International Conference on Machine Vision

Applications, pp. 22-25. 2015. DOI:10.1109/MVA.2015.7153124.
[16] J. Heo, et al. Productive high - complexity 3D city modeling with point clouds collected from terrestrial LiDAR. Computers, Environment and Urban Systems 41:26-38, 2013. DOI:10.1016/j.compenvurbsys.2013.04.002

[17] X. Xiong, et al. Automatic creation of semantically rich $3 \mathrm{D}$ building models from laser scanner data. Automation in Construction 31:325-337, 2013. DOI:10.1016/j.autcon.2012.10.006

[18] H. Woo, et al. A new segmentation method for point cloud data. International Journal of Machine Tools and Manufacture 42(2):167-178, 2002. DOI:10.1016/S0890-6955(01)00120-1

[19] T. Rabbani. Automatic reconstruction of industrial installations using point clouds and images. Tech. rep., Civil Engineering and Geosciences, 2006.

[20] J. Jung, et al. Automated 3D volumetric reconstruction of multiple-room building interiors for as-built BIM. Advanced Engineering Informatics 38:811-825, 2018. DOI:10.1016/j.aei.2018.10.007.

[21] R. Ambrus, et al. Automatic room segmentation from unstructured 3-D data of indoor environments. IEEE Robotics and Automation Letters 2(2):749-756, 2017. DOI:10.1109/LRA.2017.2651939

[22] S. Ochmann, et al. Automatic reconstruction of parametric building models from indoor point clouds. Computers $\&$ Graphics 54:94-103, 2016. DOI:10.1016/j.cag.2015.07.008

[23] Q. Huang, et al. Recurrent slice networks for 3D segmentation on point clouds. In IEEE Conference on Computer Vision and Pattern Recognition, pp. 2626-2635. 2018. DOI:10.1109/CVPR.2018.00278

[24] B. Yang, et al. Semiautomated building facade footprint extraction from mobile LiDAR point clouds. IEEE Geoscience and Remote Sensing Letters 10:766-770, 2013. DOI:10.1109/LGRS.2012.2222342. 